\title{
Prophylaxis of pyelonephritis by aminoglycosides accumulated in the kidney
}

\author{
J. Bille and M. P. Glauser
}

\begin{abstract}
Division des Maladies Infectieuses, Départment de Médecine, Centre Hospitalier Universitaire Vaudois, Lausanne, Switzerland
\end{abstract}

\begin{abstract}
When given prophylactically, gentamicin accumulates and persists in the rat kidney and affords protection against obstructive acute Escherichia coli pyelonephritis. Similar protection is observed after administration of amikacin, netilmicin and tobramycin, which accumulate to various degrees in the renal parenchyma. In those animals developing pyelonephritis despite aminoglycoside prophylaxis, renal infection and inflammation are reduced during the acute phase of the disease. This results in almost complete protection against renal scarring later on.
\end{abstract}

Administrée à titre prophylactique, la Gentamicine s'accumule dans le rein du rat et le protège contre l'apparition de pyélonéphrite aiguë à $E$. coli. Un effet protecteur identique est observé après administration d'Amikacine, de Nétilmicine, et de Tobramycine, qui s'accumulent à des degrés divers dans le parenchyme rénal.

Chez l'animal, lorsqu'apparaît une pyélonéphrite, malgré le traitement prophylactique par les aminoglycosides, l'infection et l'inflammation rénales sont diminuées durant la phase aiguë de la maladie. Ceci réduit le risque de lésion et de formation ultérieure de tissu cicatriciel.

\section{Introduction}

The accumulation and persistence of aminoglycoside antibiotics in the renal parenchyma has been reported in man and in animals. Although often considered to be responsible for the nephrotoxicity of these antibiotics, these pharmacokinetic properties have rarely been used to a therapeutic advantage.

In a rat model of ascending pyelonephritis (Brooks, Lyons \& Braude, 1974), we determined if persistence of gentamicin in the kidney can prevent the occurrence of pyelonephritis and we compared the prophylactic activity of different aminoglycosides against the development of pyelonephritis.

\section{Material and Methods}

\section{Production of pyelonephritis}

Pyelonephritis was produced by injecting into the bladder a $1-\mathrm{ml}$ inoculum of broth

Address for correspondence and reprints: Dr M. P. Glauser, Division of Infectious Diseases, Department of Internal Medicine, CHUV-1011 Lausanne, Switzerland. 
containing $10^{3}$ of Escherichia coli. Reflux into both ureters occurred consistently when urine appeared at the external urethral meatus. A silk ligature was then loosely tied around the left ureter, creating partial obstruction. The ligature was left in place for $18 \mathrm{~h}$, and then carefully removed from the outside. During the obstructive phase, infection developed above the ligature and spread to the left kidney, provoking an acute suppurative pyelonephritis.

Three days after infection, the kidney was enlarged and had numerous small abscesses on its surface, spreading into the papilla. The most reliable measure of the degree of inflammation in the left kidney was its weight. During the acute phase, it increased in proportion to the inflammation, whereas its decline in the chronic phase reflected the amount of kidney destruction. In order to minimize the variation of kidney weights between animals, the ratio of the weight of the left pyelonephritic kidney to that of the right non-pyelonephritic kidney weight was used (Glauser, Lyons \& Braude, 1978). This ratio is 1 in normal rats. The grade of infection was expressed by the logarithm of the number of cfu per gram of kidney.

\section{Prophylaxis of acute pyelonephritis with gentamicin}

The first series of experiments was designed to test whether gentamicin would prevent pyelonephritis if given before operation.

Four groups of rats received gentamicin intra-muscularly as follows:

the first group received 1 injection of $4 \mathrm{mg} / \mathrm{kg}$ of gentamicin $72 \mathrm{~h}$ before operation;

the second group received 3 injections of gentamicin at $12 \mathrm{~h}$ intervals, the last injection $72 \mathrm{~h}$ before operation;

the third group received 7 injections of gentamicin at $12 \mathrm{~h}$ intervals, the last injection $18 \mathrm{~h}$ before operation.

The control group received saline only.

Prophylaxis of acute and chronic pyelonephritis with amikacin, gentamicin, netilmicin or tobramycin

Groups of animals received 3 intramuscular doses of aminoglycoside at 12-h intervals, the last dose $72 \mathrm{~h}$ before operation. Control rats received 3 injections of saline. The animals were killed 3 days after operation in the acute pyelonephritis experiments, or 3 months later in the chronic pyelonephritis experiments. The doses of gentamicin and tobramycin were $4 \mathrm{mg} / \mathrm{kg}$, of $20 \mathrm{mg} / \mathrm{kg}$ for amikacin and of $5 \mathrm{mg} / \mathrm{kg}$ for netilmicin.

The strain of $E$. coli used for infecting the rats was inhibited by $2 \mu \mathrm{g}$ of gentamicin $/ \mathrm{ml}, 2 \mu \mathrm{g}$ of tobramycin $/ \mathrm{ml}, 4 \mu \mathrm{g}$ of amikacin $/ \mathrm{ml}$ and $1 \mu \mathrm{g}$ of netilmicin $/ \mathrm{ml}$. The levels of aminoglycosides were measured in the kidney homogenates by the agar diffusion method diluting known amounts of the aminoglycosides in normal kidney homogenate as standards.

\section{Kidney tissue levels of prophylactic aminoglycosides}

In order to determine the amount of antibiotics that had accumulated and persisted in the kidney parenchyma, different groups of rats were killed 3 days after completion of the aminoglycoside prophylaxis, at the time experimental rats were operated on and infected. 


\section{Results}

\section{Prophylaxis of acute pyelonephritis with gentamicin}

Thirteen out of the 17 control rats receiving prophylactic saline (76\%) suffered severe unilateral acute pyelonephritis. The same incidence $(22 / 28,79 \%)$ was seen after 1 single injection; but only $20 \%(4 / 20)$ of the rats challenged $72 \mathrm{~h}$ after the last of 3 injections showed evidence of pyelonephritis. In the last group, among 8 rats infected $12 \mathrm{~h}$ after the last of 7 injections, none of the kidneys showed pyelonephritis.

The question then arose as to whether the low incidence of pyelonephritis seen $72 \mathrm{~h}$ after 3 injections was due to urine levels of gentamicin sufficient to kill the infecting inoculum during the partial obstruction. In this group of rats receiving 3 injections, the pelvic fluid was cloudy at the time of removal of the partial obstruction, and contained a mean of $10^{7}$ viable $E$. coli $/ \mathrm{ml}$. In addition no measurable antibiotic activity was detected by bioassay at this time in the urines.

Thus in these experiments gentamicin accumulation retained very effective antimicrobial activity in the rat kidney parenchyma and prevented pyelonephritis when given before infection. It is widely accepted that in patients, antimicrobial drugs must reach adequate urinary concentrations in order to sterilize the urinary infection that might be responsible for kidney involvement. These results in the rat suggest that accumulation and persistence of gentamicin in the kidney parenchyma can prevent its infection in the absence of antibacterial activity in the urine.

\section{Prophylaxis of acute and chronic pyelonephritis with amikacin, gentamicin, netilmicin or tobramycin}

In comparison with gentamicin, the newer aminoglycoside antibiotics accumulate to a lesser extent in the renal tissue and might display a different degree of protection in this animal model of pyelonephritis.

The second series of experiments compared the effect of four aminoglycosides given prophylactically on the incidence and on the severity of acute and chronic pyelonephritis.

The incidence of pyelonephritis (acute and chronic) was reduced from 61\% $(78 / 128)$ in control rats to $33 \%(20 / 60, P<0.001)$ in rats receiving intramuscular prophylactic amikacin, to $25 \%(16 / 65, P<0.001)$ after intramuscular gentamicin, to $36 \%(19 / 53, P<0.001)$ after intramuscular netilmicin, and to $29 \%(15 / 51$, $P<0.001)$ after intramuscular tobramycin.

Again this protection was conferred even though at the time of operation no residual antimicrobial activity in the serum was detected by bioassay, and only traces of antibiotics were present in the urine, well below the MICs for the 4 aminoglycoside antibiotics for the infecting $E$. coli. Protection was therefore due to the antimicrobial activity of the accumulated and persistent aminoglycosides in the kidney parenchyma.

This protection was not complete and between a quarter and a third of the animals given prophylactic aminoglycosides developed pyelonephritis.

Table I analyses the severity of inflammation during acute pyelonephritis and of subsequent parenchymal destruction during chronic pyelonephritis, as expressed by the ratio of the left kidney weight to the right kidney weight. As can be seen, there was a marked reduction of inflammation in the four groups of rats developing pyelonephritis despite aminoglycoside prophylaxis. This reduction of inflammation 
Table I. Severity of acute and chronic pyelonephritis as expressed by the kidney weight ratio after prophylactic aminoglycoside antibiotics

\begin{tabular}{|c|c|c|}
\hline \multirow{2}{*}{ Group of rats } & \multicolumn{2}{|c|}{ Kidney weight ratio (left to right) } \\
\hline & acute pyeionephritis & chronic pyelonephritıs \\
\hline Controls & $1.97 \pm 0.3$ & $0.56 \pm 0.3$ \\
\hline Amikacin & $1 \cdot 60 \pm 0.3^{*}$ & $0.89 \pm 0.1^{*}$ \\
\hline Gentamicin & $1.57 \pm 0.2^{*}$ & $0.96 \pm 0.1^{*}$ \\
\hline Netilmicin & $1 \cdot 57 \pm 0.2^{*}$ & $0.88 \pm 0.1^{*}$ \\
\hline Tobramycin & $1 \cdot 40 \pm 0 \cdot 2^{*}$ & $0.93 \pm 0.1^{*}$ \\
\hline
\end{tabular}

$* P<0.001$ when compared to controls.

Table II. Severity of infection during acute and chronic pyelonephritis after prophylactic aminoglycoside antibiotics

\begin{tabular}{lcc}
\hline Group of rats & \multicolumn{2}{c}{$\log _{10}$ cfu/g of kidney } \\
chronic pyelonephritis
\end{tabular}

* $P<0.001$ when compared to controls.

during the acute phase of the disease was followed by a striking reduction in the extent of parenchymal destruction 3 months later compared with the destruction observed in controls.

Table II analyses the severity of infection during acute and chronic pyelonephritis as expressed by the number of CFU of $E$. coli recovered from kidney homogenates. During the acute phase of pyelonephritis, there was a significant reduction of infection in the four groups of rats receiving prophylactic antibiotics. However this reduction was not found 3 months after prophylaxis.

Kidney tissue levels of prophylactic aminoglycosides

The renal tissue level of gentamicin $(71 \pm 19 \mu \mathrm{g} / \mathrm{g}$ of kidney tissue) was significantly higher than the level of amikacin $(44 \pm 13 \mu \mathrm{g} / \mathrm{g})$ of netilmicin $(43 \pm 22 \mu \mathrm{g} / \mathrm{g})$ and of tobramycin $(24 \pm 15 \mu \mathrm{g} / \mathrm{g})$. Amikacin, which was given in a dosage five times greater than gentamicin and tobramycin, did not show a corresponding increase in the degree of accumulation. Its relative accumulation was therefore the least of all four aminoglycosides studied.

\section{Discussion}

Rats given 7 prophylactic doses of gentamicin, the last dose only $12 \mathrm{~h}$ before induction of pyelonephritis, displayed complete protection against kidney infection and suppuration. This protection was probably due to there being sufficient antibiotic in the urine to kill the infecting inoculum. In contrast, protection was still important, but not complete when aminoglycosides were reduced to 3 doses, the last dose given $72 \mathrm{~h}$ before the induction of pyelonephritis. This protection was 
conferred in the absence of detectable active antibiotic in the urire, and could therefore be attributed to antibiotic stored in the renal parenchyma. Protection against pyelonephritis was observed despite the presence of $10^{7} \mathrm{bacteria} / \mathrm{ml}$ in pelvic urine and in the presence of obstruction. It has been shown (Stamey, Govan \& Palmer, 1965) that to sterilize the urine of infected patients with kidney involvement, it is sufficient that antimicrobial drugs reach adequate urinary concentrations. The experiments presented here show that aminoglycosides persisting in the kidney prevent obstructive pyelonephritis even if there is not enough antimicrobial activity in the urine to abolish heavy pelvic infection.

In the experiments that compared the prophylactic activity of 3 doses of four aminoglycosides, one could have expected complete protection, if one correlate the levels of each aminoglycoside achieved in the kidney parenchyma at the time of operation and the MICs of the test organism. These levels, however, were measured in whole kidney homogenates and it is well established that aminoglycosides accumulate to a lesser extent in the medulla and papilla of the kidney where ascending pyelonephritis develops, than in the cortex (Luft et al., 1978). Furthermore, accumulated aminoglycosides are mostly located inside the tubular cells, bound to cell organelles, whereas infection starts in the interstitium. Lastly the high concentrations of urea and other solutes in the papilla and medulla decrease the antibacterial activity of aminoglycosides in the kidney. For these reasons at least, the levels of aminoglycosides as measured in these experiments did not correlate with complete in-vivo protection against the development of pyelonephritis. The antibacterial activity of the accumulated aminoglycosides in the kidney parenchyma was not sufficient to suppress acute pyelonephritis completely, as was achieved when the antibiotics were given $12 \mathrm{~h}$ before infection.

Those animals that did develop pyelonephritis despite aminoglycoside prophylaxis had less severe acute kidney infection and inflammation. This resulted 3 months later in almost complete protection against renal scarring.

Previous experiments have suggested that the formation of kidney scars results less from persisting infection than from suppurative necrosis during the acute phase of pyelonephritis (Glauser et al., 1978). In the present experiments, next to diminished infection, aminoglycoside prophylaxis afforded significant reduction of inflammation in those treated animals that developed acute pyelonephritis reflected 3 months later by a striking reduction of kidney destruction. This observation underlines the critical role of acute inflammation in the development of parenchymal damage.

\section{References}

Brooks, S. J. D., Lyons, J. M. \& Braude, A. I. (1974). Immunization against retrograde pyelonephritis. I. Production of an experimental model of severe ascending $E$. coli pyelonephritis without bacteremia in rats. American Journal of Pathology 74, 345-58.

Glauser, M. P., Lyons, J. M. \& Braude, A. I. (1978). Prevention of chronic experimental pyelonephritis by suppression of acute suppuration. Journal of Clinical Investigation 61 , 403-7.

Luft, F. C., Bloch R., Sloan, R. S., Yum, M. N., Costello, R. \& Maxwell, D. R. (1978). Comparative nephrotoxicity of aminoglycoside antibiotics in rats. Journal of Infectious Diseases 138, 541-5.

Stamey, T. A., Govan, D. E. \& Palmer J. M. (1965). The localization and treatment of urinary tract infections: the role of bactericidal urine levels as opposed to serum levels. Medicine 44, 1-36. 